\title{
Percepções da Dor nas Pacientes com Câncer de Mama
}

\author{
Perceptions of Pain in Breast Cancer Patients \\ Percepciones del Dolor en Pacientes con Cáncer de Mama
}

Recebido: 08/06/2021 | Revisado: 16/06/2021 | Aceito: 21/06/2021 | Publicado: 23/06/2021

\author{
Gabriel Antonio Meireles \\ ORCID: https://orcid.org/0000-0001-8141-2231 \\ Centro Universitário Celso Lisboa, Brasil \\ E-mail: enf.gabrielantonio@gmail.com \\ Jorge Leandro do Souto Monteiro \\ ORCID: https://orcid.org/0000-0003-1705-7620 \\ Centro Universitário Celso Lisboa, Brasil \\ E-mail: jorgeleandromonteiro@gmail.com \\ Fernanda Souza Camara \\ ORCID: https://orcid.org/0000-0003-1922-5966 \\ Centro Universitário Celso Lisboa, Brasil \\ E-mail: soouza.fernanda@gmail.com \\ Rosana Fidelis Coelho Vieira \\ ORCID: https://orcid.org/0000-0001-7509-9402 \\ E-mail: rosanafidelis72@gmail.com \\ Claudia Maria Teixeira Palhota Menezes \\ ORCID: https://orcid.org/0000-0001-6379-634X \\ E-mail: palhotamenezes@gmail.com
}

Instituto Nacional de Câncer José Alencar Gomes da Silva, Brasil

Instituto Nacional de Câncer José Alencar Gomes da Silva, Brasil

\begin{abstract}
Resumo
Objetivo: analisar em publicações científicas evidências que descrevam a percepção da dor de mulheres portadoras de câncer de mama. Metodologia: trata-se de um estudo descritivo realizado através de uma revisão integrativa, com abordagem qualitativa e análise de conteúdo de Bardin, a luz do referencial teórico de Cicely Saunders. No percurso metodológico do processo de categorização, para escolha das categorias adotamos o critério semântico que preconiza a seleção a partir de temas. Resultados: a amostra foi composta por 14 artigos, classificados em níveis de evidência segundo o Oxford Centre for Evidence-Based Medicine, onde 3 estudos (21,43\%) foram classificados como alto nível de evidência, pesquisas com nível moderado representaram 9 dos artigos $(64,28 \%)$ e com nível de evidência baixo para a prática clínica, 2 artigos (14,28\%). Conclusão: o presente estudo demonstrou um destaque para a valorização da dor física, tal fato, é um retrato de uma cultura de cuidado amparado no modelo biomédico e na medicalização, baseado nisso, a dor emocional/mental e social/familiar foram menos contextualizadas, ao passo que a dor espiritual não foi citada, levando-nos a refletir acerca do conceito de dor total, que não deveria ser negligenciado em mulheres portadoras de câncer de mama.
\end{abstract}

Palavras-chave: Câncer de mama; Manejo da dor; Percepção da dor.

\begin{abstract}
Objective: to analyze in scientific publications evidence that describes the perception of pain in women with breast cancer. Methodology: this is a descriptive study carried out through an integrative review, with a qualitative approach and content analysis by Bardin, in the light of the theoretical framework of Cicely Saunders. In the methodological path of the categorization process, to choose the categories we adopted the semantic criterion that advocates the selection based on themes. Results: the sample consisted of 14 articles, classified in levels of evidence according to the Oxford Center for Evidence-based Medicine, where 3 studies (21.43\%) were classified as high level of evidence, research with moderate level represented 9 of the articles (64.28\%) and with a low level of evidence for clinical practice, 2 articles (14.28\%). Conclusion: the present study demonstrated a highlight for the valorization of physical pain, this fact is a portrait of a culture of care supported by the biomedical model and medicalization, based on that, emotional / mental and social / family pain was less contextualized, whereas spiritual pain was not mentioned, leading us to reflect on the concept of total pain, which should not be neglected in women with breast cancer.
\end{abstract}

Keywords: Breast cancer; Pain management; Pain perception.

\section{Resumen}

Objetivo: analizar en publicaciones científicas evidencia que describa la percepción del dolor en mujeres con cáncer de mama. Metodología: se trata de un estudio descriptivo realizado a través de una revisión integradora, con enfoque cualitativo y análisis de contenido por Bardin, a la luz del marco teórico de Cicely Saunders. En la trayectoria 
metodológica del proceso de categorización, para elegir las categorías se adoptó el criterio semántico que aboga por la selección basada en temas. Resultados: la muestra estuvo conformada por 14 artículos, clasificados en niveles de evidencia según el Oxford Center for Evidence-based Medicine, donde 3 estudios $(21.43 \%)$ fueron clasificados como de alto nivel de evidencia, la investigación con nivel moderado representó 9 de los artículos $(64,28 \%)$ y con bajo nivel de evidencia para la práctica clínica, 2 artículos (14,28\%). Conclusión: el presente estudio demostró un destaque para la valorización del dolor físico, este hecho es un retrato de una cultura del cuidado sustentada en el modelo biomédico y la medicalización, en base a que, el dolor emocional / mental y social / familiar fueron menos contextualizados, mientras que no se mencionó el dolor espiritual, lo que nos llevó a reflexionar sobre el concepto de dolor total, que no debe descuidarse en las mujeres con cáncer de mama.

Palabras clave: Cáncer de mama; Manejo del dolor; Percepción del dolor.

\section{Introdução}

O Instituto Nacional do Câncer [INCA] (2020a), aponta que o câncer de mama é o segundo câncer mais incidente no Brasil, tendo a estimativa de 66.280 novos casos em 2020. De uma perspectiva internacional, o relatório global sobre o câncer, who report on cancer, setting priorities, investing wisely and providing care for all, divulgado em fevereiro de 2020 pela Organização Mundial da Saúde, salienta que em 2018 a incidência de casos de câncer de mama foi de 2.088.849 (WHO, 2020).

Comparando-se aos outros tipos de câncer com grande potencial de incidência, segundo a estimativa do INCA para o ano de 2020, observa-se que os homens são mais acometidos pelos tumores malignos de próstata (29,2\%), consideravelmente, seguido das neoplasias no cólon e reto $(9,1 \%)$; pulmão $(7,9 \%)$; estômago $(5,9 \%)$ e cavidade oral $(5,0 \%)$. Enquanto nas mulheres percebe-se uma grande incidência sobre o câncer de mama (29,7\%), seguidamente do câncer de cólon e reto (9,2\%); câncer de colo do útero (7,5\%); pulmão $(5,6 \%)$ e tireoide $(5,4 \%)$. Entretanto, para ambos os sexos o câncer de pele não melanoma é o que possui o maior número de casos. Assim sendo, a incidência de casos novos de câncer para cada ano do triênio 2020-2022, será de 83.770 em homens e de 93.170 em mulheres (INCA, 2020b).

O carcinoma de mama é um dos mais diagnosticados em todo o mundo, tendo grande importância na saúde das mulheres. Entretanto, também afeta a população masculina. De acordo com Araújo et al. (2018), a neoplasia mamária masculina é rara, representando menos de $1 \%$ dos casos de câncer de mama, mas têm demonstrado índices crescentes de incidência.

Segundo Santana, Rippel e Fortes (2020), no boletim informativo para prevenção e diagnóstico precoce, o câncer de mama é descrito como uma doença multifatorial. Portanto, os diversos fatores que influenciam o aumento dos riscos da doença são: idade, menstruação precoce, menopausa tardia, uso de contraceptivos orais e terapia de reposição hormonal pós menopausa, sobrepeso/obesidade, consumo excessivo de álcool, inatividade física e casos de câncer de mama na família. O mesmo documento destaca que cerca de $30 \%$ dos cânceres de mama podem ser evitados com a adoção de hábitos saudáveis como: prática de atividade física, alimentar-se bem, e entre outros, manter o peso corporal equilibrado.

Salienta-se ainda que os principais sinais e sintomas de câncer de mama são nódulos na mama e/ou axila, dor mamária e alterações da pele que recobre a mama, como abaulamentos ou retrações com aspecto semelhante à casca de laranja (Santana et al., 2020). Informações relevantes, que devem ser disseminadas para as mulheres, com objetivo de aumentar a suspeição e a detecção precoce, contribuindo assim para o diagnóstico.

Couto, Guerra, Firme e Teixeira (2017), evidenciam que ao contrário do que ocorre em boa parte dos países desenvolvidos, que apresentam tendência de aumento da incidência e de redução da mortalidade por câncer de mama, no Brasil a taxa de mortalidade por esse tipo de câncer cresceu nas últimas três décadas. Nessa perspectiva, os pacientes oncológicos vivenciam o sofrimento ocasionado pela doença, mas em países menos desenvolvidos os determinantes sociais e as iniquidades acentuam a angústia e influenciam diretamente as taxas de mortalidade. Logo, a criação de políticas públicas que tornem o itinerário terapêutico menos sofrido, objetivando a chegada nos centros de referência em menor tempo é fundamental.

Isto posto, os protocolos da atenção básica destinados a abordar a saúde das mulheres no Brasil, preconizam o rastreamento de câncer de mama com a mamografia a cada dois anos para mulheres entre 50 e 69 anos. Essa importante política 
pública consiste na realização de testes ou exames diagnósticos em populações ou pessoas assintomáticas, com a finalidade de diagnóstico precoce, para reduzir a morbidade e mortalidade da doença (Brasil, 2016).

Sob o mesmo ponto de vista, os protocolos da atenção primária preconizam outras ações primordiais de prevenção e acompanhamento dos casos, tais como: intervenção sobre os fatores de risco modificáveis para o câncer de mama; auxílio na reabilitação, retorno às atividades e a reinserção na comunidade; orientação de cuidados; acompanhamento clínico e o controle da doença; orientação quanto aos direitos dos portadores de câncer (Brasil, 2016).

Quando uma mulher é diagnosticada com câncer de mama, ela pode se deparar com um sofrimento que assume diversos aspectos, como por exemplo, o impacto emocional do diagnóstico e passar por um tratamento debilitante. Dentre estes destacase a vivência da dor que se torna constante e incapacitante.

Em 2020, a Associação Internacional para Estudos da Dor (IASP), reuniu 14 membros com ampla experiência em ciência clínica relacionada a dor, para compor o Subcomitê de Taxonomia e construir um novo conceito. Ao contrário do concebido em 1979, a nova definição entende o assunto em toda a sua amplitude: "A dor é uma experiência sensitiva e emocional desagradável, associada, ou semelhante àquela associada, a uma lesão tecidual, real ou potencial” (Raja et al., 2020, p.7).

Desse modo, a demonstração de uma concepção mais integral é evidenciada nas notas emitidas pelo conselho da IAPS sobre a definição de 2020: a dor é sempre uma experiência individual influenciada por fatores biológicos, psicológicos e sociais; através das suas experiências de vida, as pessoas aprendem o conceito de dor; o relato de uma pessoa sobre uma experiência de dor deve ser respeitado; embora a dor geralmente cumpra um papel adaptativo, ela pode ter efeitos adversos na função e no bemestar social e psicológico; a descrição verbal é apenas um dos vários comportamentos para expressar a dor; a incapacidade de comunicação não invalida a possibilidade de um ser humano ou um animal sentir dor (Raja et al., 2020, p.7).

No paciente oncológico, a dor está relacionada com a neoplasia em 60-90\% dos casos (invasão ou compressão de estruturas), em 5-20\% dos casos está relacionado com o tratamento (radioterapia, fibrose, neuropatia pós-quimioterapia, pósoperatório) e em 3-10 \% a dor não tem relação com a doença neoplásica. Ademais, a dor é comum, principalmente para aqueles que são expostos a procedimentos invasivos, não é simples para o profissional a avaliação da experiência dolorosa, pois envolve fatores multidimensionais (Costa, Santos, Alves \& Costa, 2007; Oliveira, Roque \& Maia, 2019).

Mediante o exposto percebe-se a necessidade em analisar a percepção da dor em mulheres com câncer de mama e como isso pode influenciar o cuidado e o cotidiano dessas mulheres. Delimitamos como objetivo da presente pesquisa, analisar em publicações científicas evidências que descrevam a percepção da dor de mulheres portadoras de câncer de mama. Assim, realizamos um levantamento em fontes nacionais e internacionais, a fim de descrever sob a análise de conteúdo o objeto de estudo através de uma abordagem qualitativa.

\section{Metodologia}

Trata-se de um estudo descritivo, realizado através de uma revisão integrativa, com abordagem qualitativa, utilizando para o tratamento dos dados a análise de conteúdo de Bardin. Além disso, os dados foram discutidos a partir do referencial teórico de Cicely Saunders.

Nesse sentido, a análise de conteúdo, segundo Bardin é definida como um conjunto de técnicas de análise das comunicações visando obter, por procedimentos sistemáticos e objetivos de descrição do conteúdo das mensagens, indicadores que permitam a inferência de conhecimentos relativos às condições de produção e recepção destas mensagens. A análise de conteúdo atualmente é um conjunto de instrumentos metodológicos cada vez mais sutis em constante aperfeiçoamento. O fator comum destas técnicas varia, desde o cálculo de frequências que fornece dados cifrados, até a extração de estruturas traduzíveis em modelos - é uma hermenêutica controlada, baseada na dedução: a inferência (Bardin, 2011).

Para tanto, a revisão integrativa foi utilizada como método de seleção e organização dos achados nas bases de dados, 
antes da aplicação da análise de conteúdo de Bardin. A revisão integrativa é um método específico, que fornece uma compreensão mais abrangente de um determinado fenômeno, contribuindo de forma significante para a prática baseada em evidências, pois, permite a incorporação dos achados na prática clínica (Sousa, Vieira, Severino \& Antunes, 2017).

Este método requer a formulação de um problema, uma pesquisa de literatura, a avaliação crítica de um conjunto de dados, a análise de dados e, a apresentação dos resultados. Deste modo, permite reunir e sintetizar resultados de pesquisas sobre um tema delimitado ou questão, de forma sistemática e ordenada (Sousa et al., 2017).

Portanto, mediante ao problema de pesquisa: “Qual a percepção da dor de mulheres com câncer de mama?”, foi utilizada a estratégia PICo (Participantes ou população, Fenômeno de interesse e contexto do estudo), como participantes evidenciou-se: mulheres; câncer de mama; o fenômeno de interesse foi: dor no câncer de mama; o contexto do estudo convergiu para: percepção e manejo da dor, tanto pelas mulheres quanto pelos profissionais.

Posteriormente, após o mapeamento dos termos no DeCs e MeSH, uma busca foi realizada nas seguintes bases de dados: LILACS, MEDLINE, Web of Science, Scopus e CINAHL. A estratégia de busca utilizada se deu a partir da conjugação de operadores booleanos, conforme demonstrado na Tabela 1, tal qual o número de artigos recuperados em cada base na busca inicial, que soma ao todo 57 artigos.

Tabela 1. Estratégias de busca nas bases de dados selecionadas. Rio de Janeiro, RJ, Brasil, 2020. (continua)

\begin{tabular}{ccc}
\hline BASES DE DADOS & ESTRATÉGIAS & RECUPERADOS \\
\cline { 2 - 3 } & tw:( ("neoplasias da mama” OR “carcinoma de mama” OR "Câncer de mama" \\
BVS/ LILACS & OR “carcinoma mamário humano” OR “tumor maligno da mama”) AND \\
(manejo da dor) AND (percepção OR percepções)) & 5 \\
\hline & (("Breast Neoplasms"[Title/Abstract] OR "Breast Tumor"[Title/Abstract] OR \\
& "Human Mammary Carcinomas" "Breast Tumors"[Title/Abstract] OR "Breast \\
PUBMED/ MEDLINE & Cancer"[Title/Abstract] OR "Mammary Cancer"[Title/Abstract]) AND ("Pain \\
& Management"[Title/Abstract] OR "Pain Managements"[Title/Abstract])) \\
& AND (Perception[Title/Abstract] OR Perceptions[Title/Abstract])
\end{tabular}

Tabela 1. Estratégias de busca nas bases de dados selecionadas. Rio de Janeiro, RJ, Brasil, 2020. (conclusão)

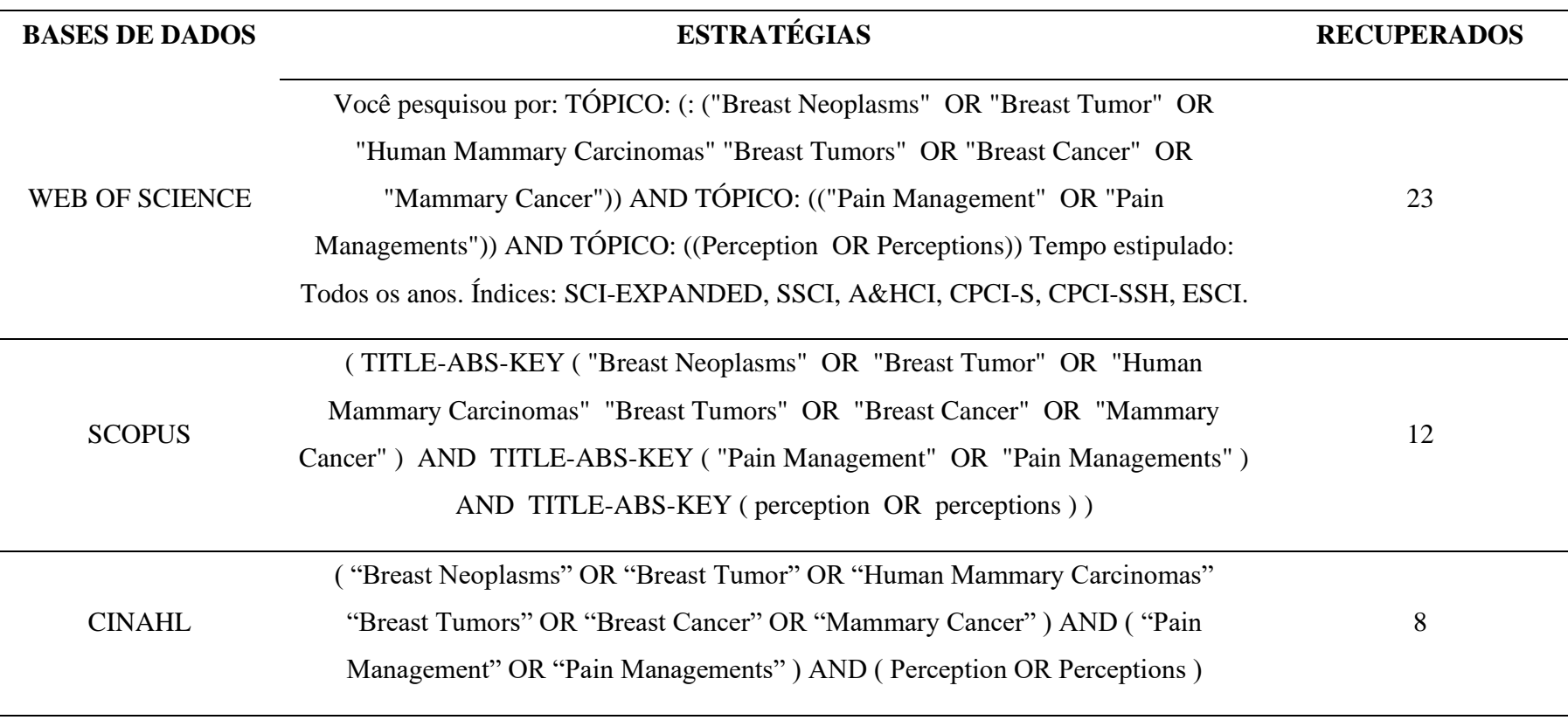


Por conseguinte, destaca-se a ausência de descritores mais específicos para o melhor delineamento do tema. Não sendo encontrados no sistema do Decs: dor total; dor espiritual; dor emocional; dor social. Para compreensão da percepção da dor optou-se por utilizar o descritor "manejo da dor".

Deste modo, após a realização das buscas, acrescentou-se como critérios para inclusão: artigos originais e de revisão disponibilizados na íntegra, gratuitamente ou de fácil acesso, com recorte temporal de 10 anos (2010 - 2020), publicados nos idiomas inglês, português e espanhol. Os critérios de exclusão foram: estudos duplicados, estudos fora da temática (câncer de mama e dor) e publicações em anais de eventos científicos.

Logo, foram selecionados 14 artigos, como ilustra a síntese da busca bibliográfica na Figura 1. Utilizamos como ferramentas de busca, armazenamento e seleção dos estudos, o EndNote Web para extração dos estudos recuperados nas bases de dados e remoção dos duplicados e o Rayyan QCRI para organização do processo de inclusão e exclusão.

Figura 1. Fluxograma do processo de seleção dos estudos. Rio de Janeiro, RJ, Brasil, 2020.

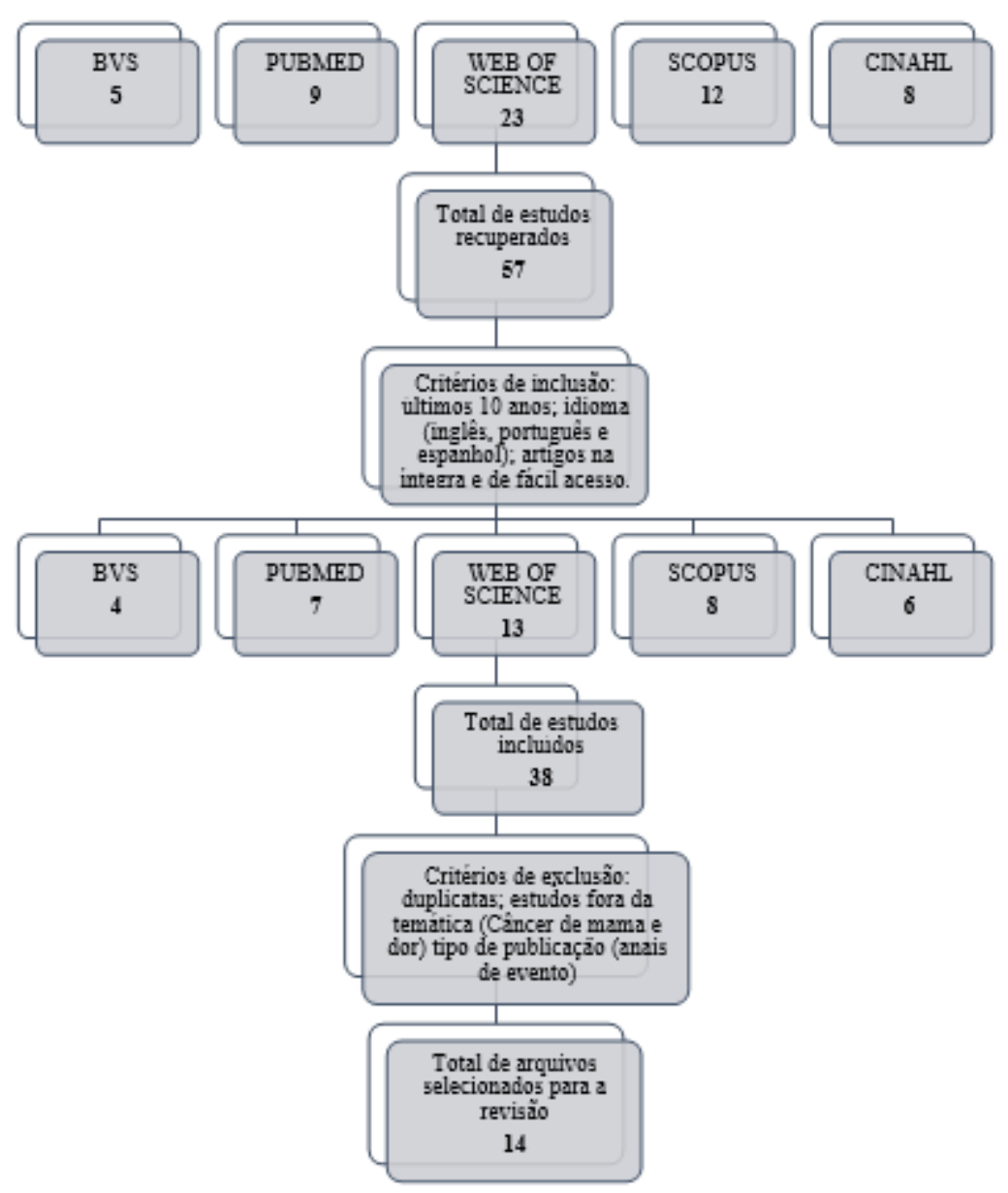

Fonte: Autores (2020).

\section{Resultados}

A amostra foi composta por 14 artigos, organizados na Tabela 2 em título, autores, periódicos, ano de publicação, metodologia e identificados por números cardinais, que possibilitaram sua ordenação em ordem crescente de acordo com o ano de publicação. Dentre os artigos incluídos na revisão, 14 (100\%), foram publicados em inglês; quanto aos países onde foram desenvolvidos, houve prevalência dos Estados Unidos da América, com 6 publicações (42,82\%); Dinamarca com 2 (14,28\%); 
Brasil, França, Holanda, Israel, Reino Unido e Suécia, com 1 artigo cada (7,15\%), características que podem ser atribuídas as bases de dados utilizadas e ao uso dos MeSH Terms.

Ademais, salienta-se que na ordem cronológica dos 14 artigos recuperados os achados se dividem em: 2010, 1 artigo (7,15\%); 2012, 2 artigos (14,28\%); 2014, 2 artigos (14,28\%); 2015, 1 artigo (7,15\%); 2017, 1 artigo (7,15\%); 2018, 3 artigos (21,43\%); 2019, 2 artigos (14,28\%); 2020, 2 artigos (14,28\%). No ano de 2018 o número de publicações excedeu os outros anos.

Tabela 2. Ordenação dos estudos incluídos na Revisão. Rio de Janeiro, RJ, Brasil, 2020. (continua)

\section{TÍTULO}

1. Anxiety, depression, and pain: differences by primary cancer

\section{AUTORES}

Fischer, D. J., et Supportive Care in

al.

Center

\section{ANO DE PUBLICAÇÃO METODOLOGIA}

2010

Estudo transversal

2. Association of fibromyalgia

characteristics in patients with non-

metastatic breast cancer and the protective

Schrier, M., et

al.
Rheumatol Int

2012

Estudo de caso-

controle role of resilience

3. Effects of Pain Controllability and Discrepancy in Social Support on Depressed Mood Among Patients with Chronic Pain
International Journal

Cho, S., et al. of Behavioral

Estudo transversal

Medicine

\section{Complementary therapy support} in cancer survivorship: a survey of

Samuel C. A., $\quad$ European Journal of

\& Faithfull S. Cancer Care
complementary and alternative medicine practitioners' provision and perception of

2014

e-survey

skills

5. $\quad$ Exercise despite pain - breast cancer patient experiences of muscle and joint pain during adjuvant chemotherapy and concurrent participation in an exercise

Andersen, C., et al.
European Journal of

Cancer Care
Estudo descritivo

2014 com abordagem fenomenológica

intervention

6. Women With Breast Cancer

Experience of Chemotherapy-Induced Pain:

Triangulation of Methods

\section{Hellerstedt-}

Borjesson, S., et

al.
2015

Cancer Nursing

Mertz, B. G., et

Pain Management

al. Nursing
2017

Estudo exploratório

Women Treated for Ductal Carcinoma In

Situ.

\begin{tabular}{lccc}
\hline 8. $\quad$ Metastatic breast cancer patients' & Tometich, D. & & \\
expectations and priorities for symptom & B., et al. & & 2018 \\
improvement & & & Estudo Transversal
\end{tabular}

9. Effects of a Hypnosis Session

Before General Anesthesia on

Amraoui, J., et

al.

JAMA network open

2018
Estudo exploratório

qualitativo
Postoperative Outcomes in Patients Who

Underwent Minor Breast Cancer Surgery 
Tabela 2. Ordenação dos estudos incluídos na Revisão. Rio de Janeiro, RJ, Brasil, 2020. (conclusão)

\begin{tabular}{|c|c|c|c|c|}
\hline TÍTULO & AUTORES & PERIÓDICO & ANO DE PUBLICAÇÃO & METODOLOGIA \\
\hline $\begin{array}{l}\text { 10. Effectiveness of A Self-Care } \\
\text { toolkit for surgical breast cancer patients in } \\
\text { a military treatment facility }\end{array}$ & $\begin{array}{l}\text { Stoerkel, E., et } \\
\text { al. }\end{array}$ & $\begin{array}{l}\text { Journal of Alternative } \\
\text { and Complementary } \\
\text { Medicine }\end{array}$ & 2018 & $\begin{array}{l}\text { Ensaio clínico } \\
\text { randomizado } \\
\text { controlado }\end{array}$ \\
\hline $\begin{array}{l}\text { 11. Surgeon perception versus } \\
\text { reality: Opioid use after breast cancer } \\
\text { surgery }\end{array}$ & $\begin{array}{c}\text { Park, K. U., et } \\
\text { al. }\end{array}$ & $\begin{array}{c}\text { Journal of Surgical } \\
\text { Oncology }\end{array}$ & 2019 & $\begin{array}{c}\text { Estudo de coorte } \\
\text { exploratório }\end{array}$ \\
\hline $\begin{array}{l}\text { 12. Perceptions of patients with } \\
\text { breast and colon cancer of the management } \\
\text { of cancer-related pain, fatigue, and } \\
\text { emotional distress in community oncology }\end{array}$ & $\begin{array}{c}\text { Smith, T. G., et } \\
\text { al. }\end{array}$ & $\begin{array}{c}\text { Journal of Clinical } \\
\text { Oncology }\end{array}$ & 2019 & e-survery \\
\hline $\begin{array}{l}\text { 13. Effect of the Pilates Method on } \\
\text { Sexual Function, Pelvic floor Muscle } \\
\text { Strength and Quality of Life of Breast } \\
\text { Cancer Survivors }\end{array}$ & $\begin{array}{c}\text { Ferraz, V. S., et } \\
\text { al. }\end{array}$ & $\begin{array}{l}\text { Rev. brasileira de } \\
\text { cancerologia }\end{array}$ & 2020 & $\begin{array}{l}\text { Ensaio clínico } \\
\text { randomizado }\end{array}$ \\
\hline $\begin{array}{l}\text { 14. Kinesiophobia contributes to pain- } \\
\text { related disability in breast cancer survivors: } \\
\text { a cross-sectional study }\end{array}$ & $\begin{array}{c}\text { Van der Gucht, } \\
\text { E., et al. }\end{array}$ & $\begin{array}{c}\text { Supportive Care in } \\
\text { Cancer }\end{array}$ & 2020 & Estudo transversal \\
\hline
\end{tabular}

Fonte: Autores (2020).

Segundo o Oxford Centre for Evidence-Based Medicine [CEBM], os diferentes níveis de evidências orientam a elaboração dos graus de recomendação de condutas da área de saúde, refletindo a tomada de decisão nos diferentes graus de recomendação. Diante disso, os artigos foram tabulados destacando os níveis de evidência a fim de demonstrar a relevância desses achados (CEBM, 2013).

A qualidade da evidência tem sido classificada em quatro níveis: alta (A), moderada (B), baixa (C), muito baixa (D). Em suma, estudos provenientes de ensaios clínicos randomizados possuem nível de evidência alta, 3 estudos (21,43\%) foram classificados por esse parâmetro. Desse modo, pesquisas com nível moderado são formadas de estudos como os transversais, coorte, caso-controle, caracterizando-se como estudos observacionais, o que representa 9 dos artigos (64,28\%) (CEBM, 2013).

Além disso, os estudos de caso ou que apresentem uma série de casos, possuem um nível de evidência baixo para a prática clínica, na realidade do presente estudo, 2 artigos (14,28\%) se enquadram nessa categoria. Por último, nenhum estudo selecionado para revisão foi classificado com nível de evidência muito baixo, o que está relacionado com a informação baseada somente na opinião de especialistas (CEBM, 2013). Os graus de recomendação e os estudos classificados em cada nível são especificados na Figura 2. 
Figura 2. Artigos classificados em nível de evidência e grau de recomendação segundo o Oxford Centre for Evidence-Based Medicine. Rio de Janeiro, RJ, Brasil, 2020.

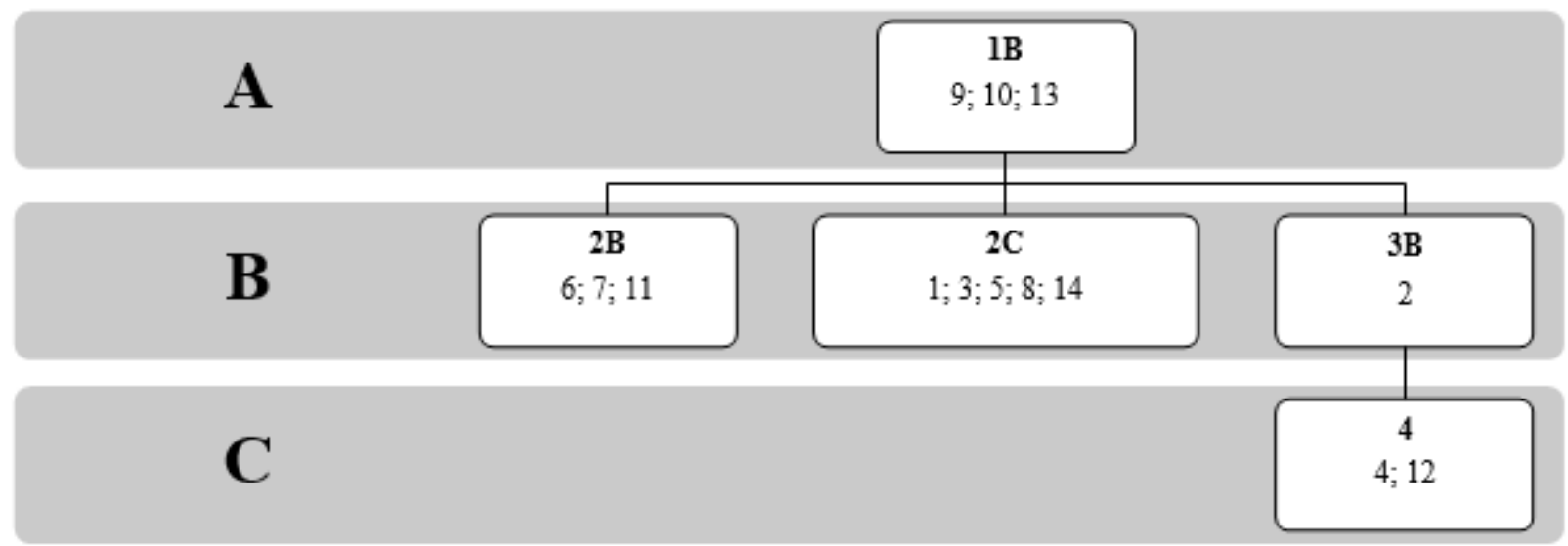

Fonte: Autores (2020).

\section{Discussão}

As etapas do desenvolvimento da análise de conteúdo de Bardin foram fundamentadas na pré-análise, que resultou na constituição do corpus e na preparação do material; posteriormente realizou-se a exploração do material selecionando-o em unidades de contexto e eixos temáticos, para categorização (Bardin, 2011).

Aprofundando-se no percurso metodológico do processo de categorização, para escolha das categorias adotamos o critério semântico que preconiza a seleção a partir de temas. As categorias iniciais configuraram-se como as primeiras impressões acerca da realidade estudada. Vale ressaltar que cada categoria foi constituída pelos trechos levantados nos 14 artigos incluídos neste estudo.

Nesse sentido, 37 categorias iniciais forneceram suporte para as categorias intermediárias, originando inicialmente as 9 categorias intermediárias, porém, ainda explorando os artigos e analisando as categorias, a constituição final foi formada por 3 categorias, denominadas "medidas de mitigação da dor física associada ao câncer de mama e ao tratamento oncológico"; “dor emocional e os impactos do autocuidado e valorização da subjetividade" e "frustração relacionada à perda dos papéis sociais e a contribuição da rede de apoio no enfrentamento da dor social”, como exposto na Tabela 3. 
Tabela 3. Processo de categorização semântica criada a partir dos 14 artigos recuperados. Rio de Janeiro, RJ, Brasil, 2020.

\section{CATEGORIAS INICIAIS}

1. Associação da dor ao tumor

\begin{tabular}{l}
\hline 2. Associação da dor ao tratamento \\
\hline 3. Relação da dor como consequência da quimioterapia \\
\hline 4. Condutas invasivas e dor intensa \\
\hline 5. Relação da cinesiofobia e incapacidade \\
\hline 6. Dificuldade em conviver com a dor \\
\hline 7. Inquietação e insônia relacionados à dor \\
\hline 8. Analgesia insuficiente \\
\hline 9. Dor persistente em todo corpo \\
\hline 10. Dor generalizada no câncer de mama \\
\hline 11. Estratégias para o controle da dor \\
\hline 12. Qualidade de vida proporcionada pelo pilates \\
\hline 13. Hipnose no pré-operatório \\
\hline
\end{tabular}

\section{CATEGORIAS INTERMEDIÁRIAS \\ Impacto do tratamento adjuvante e neoadjuvante}

14. Atividade física para aliviar a dor

15. Medo da dor e sofrimento

\begin{tabular}{l}
\hline 16. Insegurança e ansiedade \\
\hline 17. Processo de somatização \\
\hline 18. Redução da perspectiva de futuro \\
\hline 19. Diminuição da autoestima
\end{tabular}

\begin{tabular}{l} 
20. Subjetividade no enfrentamento da dor \\
\hline 21. Metaforização da dor \\
\hline
\end{tabular}

22. Catastrofização e crenças relacionadas à doença

23. Comunicação ineficaz com os profissionais de saúde

24. Resistência em procurar ajuda

25. Promoção do autocuidado

Medidas de mitigação no controle da dor

\section{CATEGORIAS FINAIS}

FREQUÊNCIA

A dor oncológica no cotidiano

(2)

I) Medidas de mitigação da dor física associada ao câncer de mama e ao tratamento oncológico
Influência do câncer nos sentimentos e emoções

\begin{tabular}{|c|c|}
\hline 26. Suporte emocional como alicerce & \multirow{4}{*}{$\begin{array}{c}\text { Rede de apoio e } \\
\text { compartilhamento da } \\
\text { experiência entre as } \\
\text { pacientes }\end{array}$} \\
\hline 27. A importância da rede de apoio & \\
\hline 28. Compartilhamento de pensamentos com outras pacientes & \\
\hline 29. Vivenciando a solitude no processo de saúde-doença & \\
\hline 30. Dificuldade de realizar qualquer atividade & \multirow{5}{*}{$\begin{array}{c}\text { Frustração } \\
\text { relacionada à perda do } \\
\text { papel social }\end{array}$} \\
\hline 31. Dor existencial associada à dependência & \\
\hline 32. Vulnerabilidade e fragilidade causadas pela dor & \\
\hline 33. Temperamento e paciência com a família afetados & \\
\hline 34. Dificuldades de comunicar a família & \\
\hline 35. Sexualidade impactada pela mastectomia & \multirow{3}{*}{$\begin{array}{c}\text { Sexualidade } \\
\text { prejudicada pela } \\
\text { doença e o tratamento }\end{array}$} \\
\hline 36. Sexualidade como tabu & \\
\hline 37. Vida sexual prejudica pela quimioterapia & \\
\hline
\end{tabular}

Fonte: Autores (2020).

Dessa forma, após definir as categorias, a última fase da análise de conteúdo de Bardin compreende o processo de interpretação e inferência dos dados (Bardin, 2011). A primeira categoria, "Medidas de mitigação da dor física associada ao câncer de mama e ao tratamento oncológico", refere-se à percepção que as mulheres com câncer de mama possuem da relação 
de causa e consequência da dor física e das formas de aliviá-la. Essa categoria tem o objetivo de descrever os aspectos que permeiam a dor física no câncer de mama.

Na segunda categoria, "Dor emocional e os impactos do autocuidado e valorização da subjetividade", discute-se os sentimentos e emoções vivenciados pelas pacientes, que são influenciadas pela subjetividade e impactam diretamente a autoestima. O objetivo dessa categoria é descrever os aspectos da dor emocional no câncer de mama.

Por conseguinte, a terceira categoria, "Frustração relacionada à perda dos papéis sociais e a contribuição da rede de apoio no enfrentamento da dor social", retrata a insatisfação das mulheres que optam pelo isolamento por se sentirem incapazes devido à dor. A finalidade dessa categoria é descrever a dor social no câncer de mama. Essas discussões serão explicitadas nas próximas subseções, cujos títulos representam as categorias que surgiram ao longo da análise de conteúdo.

Indubitavelmente, as 3 categorias possuem uma relação com a teórica Cicely Saunders, que descreveu o conceito de dor total pela primeira vez em 1964. Saunders, compreende a dor para além da apresentação física, destacando também a dor emocional/mental, social/familiar e espiritual, aspectos que se combinam e formam a complexidade do sofrimento (Saunders, 2005).

\section{Medidas de Mitigação da Dor Física Associada ao Câncer de Mama e ao Tratamento Oncológico}

As unidades de registro que contêm a reflexão relacionada a essa categoria foram as mais presentes nos dados observados, sendo mencionadas em 51 registros. Logo, essa categoria se define como a mais expressiva quantitativamente. Além disso, mediante aos artigos classificados anteriormente quanto ao nível de evidência, essa categoria possui 2 artigos com nível de evidência alta, 6 com nível moderado e 2 com nível baixo.

Muitos temem que os efeitos colaterais do tratamento sejam piores do que a própria doença. O objetivo do controle da dor física e do alívio de outros sintomas é permitir que o paciente encontre o seu corpo e suas necessidades, mesmo quando suas atividades são cada vez mais restringidas pela doença. Referindo-se à dor física, Saunders, um grande nome do cuidado paliativo moderno, ratifica que a dor pode ser totalmente aliviada na maioria e controlada satisfatoriamente no resto (Saunders, 2005).

A dor em cânceres primários é percebida por múltiplos ângulos. Um estudo que comparou tumores primários, mais especificamente, o câncer de pulmão, próstata e cabeça/pescoço, obteve como resultado uma grande proporção de sujeitos com câncer de pulmão que reportaram a origem da dor associada ao tumor, o que se diferenciou dos indivíduos com tumores de próstata e cabeça/pescoço, que atribuíram a origem da dor ao tratamento (Fischer, D. J., Villines, D., Kim, Y. O., Epstein, J. B., \& Wilkie, D. J., 2010).

Sobretudo, um achado na maioria dos estudos demonstrou que no câncer de mama a dor está mais associada ao tratamento, pois, dias após as sessões de quimioterapia as pacientes estavam convencidas de que a dor era causada principalmente pela infusão da quimioterapia. A dor vivenciada pelas mulheres tem em média uma semana de duração, com uma variação entre 6-14 dias durante os quais a intensidade da dor atingiu o pico entre 2 a 9 dias depois de um ciclo de quimioterapia (Andersen C., Rørth M., Ejlertsen B., \& Adamsen L., 2014; Hellerstedt-Börjesson, S., Nordin, K., Fja“llskog, M. L., Holmstro“m, I. K., \& Arving, C., 2015).

Consequentemente, as pacientes relataram que quando a vida começava a se normalizar, era tempo de realizar o ciclo seguinte do tratamento, um momento em que a dor atingia o seu pico e o tempo vivido se resumia a uma dor aguda com uma sensação de inquietação difícil de descrever em palavras, uma vez que não se assemelhava a nenhuma dor vivenciada nas experiências dolorosas pregressas. Dessarte, as mulheres criam descrições com ajuda de metáforas (Andersen, C. et al., 2014; Hellerstedt-Börjesson, S., et al., 2015).

Um ano após o tratamento, as dores residuais no corpo foram frequentemente atribuídas às mesmas partes do corpo como durante o tratamento, e tal fato sugeriu que a dor pode ter sido uma herança da quimioterapia. Paralelamente a isso, no 
período de 6 meses após o tratamento, as mulheres continuaram a sentir os sintomas mais prevalentes no câncer de mama (dor, fadiga e angústia). A cinesiofobia (medo do movimento) evidenciou-se como o fator mais significativo para a dor relacionada à incapacidade. Contudo, a maioria das pacientes também reportam gravidade usual moderada para fadiga, problemas no pensamento e insônia. No geral, as pacientes têm uma taxa de dor generalizada, quatro vezes maior do que uma mulher saudável que pode sentir dores naturais relacionadas ao envelhecimento (Hellerstedt-Börjesson, S., et al., 2015; Smith, T. G. et al., 2019; Van Der Gucht, E. et al., 2020).

Inegavelmente, quanto mais invasivo for o tratamento, maior será a percepção da dor e consequentemente a necessidade de utilização de opioides, com o intuito de promover analgesia. Nessa lógica, pacientes que realizaram a mastectomia e dissecção de linfonodo axilar tiveram maior uso. Entretanto, ao calcular a hipotética global anual do uso de opioides pelos pacientes, considerando a preferência de prescrição, o número de opioides não utilizados equivale a 46.165 comprimidos de hidrocodona $5 \mathrm{mg}$. Embora esse resultado demonstre a necessidade de uma vigilância quanto ao uso inadequado, a prescrição exacerbada não deve fomentar o preconceito do público, em que se acredita na perda do efeito após aumentos inevitáveis na dose, e que, na melhor das hipóteses, os pacientes ficarão sonolentos e confusos se a dor for aliviada. Essas crenças se manifestam infundadas e o ensino sobre como usar essas drogas de forma eficaz tem avançado (Andersen, C. et al., 2014; Park, K. U. et al., 2019; Saunders, 2005; Tometich, D. B. et al., 2018).

A multidimensionalidade da dor do câncer é demonstrada pelo relacionamento entre os fatores físicos e psicológicos como a depressão e ansiedade, de conformidade com os sintomas físicos e psicossociais, que são predominantes. Logo, priorizar somente as medidas farmacológicas não atende o cuidado integral. Segundo os profissionais de saúde que trabalham com a terapia holística, a prevalência dos sintomas em seus atendimentos consiste no estresse como sintoma mais frequentemente tratado; seguido por fadiga; ansiedade e depressão; insônia; gerenciamento geral da dor e linfedema (Fischer, D. J. et al., 2010; Samuel, C. A., \& Faithfull, S., 2014).

Sujeitos com câncer reinterpretam a dor usando medidas de mitigação. Exercitar-se pelo menos 3 horas por semana com atividade física (caminhando ou correndo) apresentou-se como experiência positiva para reduzir a dor, principalmente se realizada em grupo, para o desenvolvimento de uma força mental. Outrossim, o planejamento do tempo, a identificação de posições de descanso confortáveis, ou mover o corpo em uma maneira de controlar a dor também foram mencionados na literatura (Andersen, C. et al., 2014; Hellerstedt-Börjesson, S., et al., 2015; Fischer, D. J. et al., 2010).

Os achados na literatura demonstram uma influência substancial na qualidade de vida das mulheres após cirurgia de câncer de mama que realizaram sessões de Pilates. Sendo assim, os autores demonstraram a eficácia através da mensuração da percepção global da saúde, dos itens da escala funcional (funcionamento físico e emocional) e da diminuição dos itens de sintomas negativos (náuseas, dores e fadiga) (Ferraz, V. S. et al., 2020).

As medidas não farmacológicas para o controle da dor representam benefícios, baixo custo e poucos efeitos colaterais. Analogamente, o uso de hipnose antes da anestesia geral para cirurgia confirma esse achado, quando, por exemplo, um ensaio clínico aponta que em pacientes hipnotizados, o consumo de drogas como propofol e sufentanil foram menos necessárias, (200 mg) para propofol e $15 \mu \mathrm{g}$ para citrato de sufentanil. Além disso, favoreceu uma técnica de manejo das vias aéreas com uma máscara laríngea não invasiva, auxiliando também na diminuição significativa da fadiga e ansiedade na noite da cirurgia (Amraoui, J. et al., 2018; Ferraz, V. S. et al., 2020).

\section{Dor Emocional e os Impactos do Autocuidado e Valorização da Subjetividade}

Possuindo 31 registros, essa categoria se apresenta com elevada relevância ao descrever o sofrimento emocional das pacientes com câncer de mama, que está relacionado a diversos fatores. Em relação ao nível de evidência, os artigos utilizados nessa categoria se dividiram como, 1 artigo com nível de evidência alto, 7 com nível moderado e 1 com nível baixo. 
A dor não é apenas física, mas também profundamente emocional. Na verdade, a dor mental pode ser a mais intratável de todas. A ansiedade em relação à doença e seus tratamentos funde-se com a depressão evocada por capacidades cada vez menores. Desse modo, os sentimentos e emoções manifestam-se e, dentre eles, a tristeza é apropriada e deve ser enfrentada e compartilhada. Portanto, a dor emocional exige um ouvinte, em vez de apenas drogas, posto que a combinação dos dois possa ajudar a levantar uma carga inibidora e permitir que o paciente resolva problemas que pareciam incontroláveis (Saunders, 2005).

Em um estudo, com um grupo de pacientes foi demonstrado o quão importante é o papel do profissional de saúde como comunicador. Tratava-se de pacientes com carcinoma ductal in situ, uma forma inicial de neoplasia não invasivo com um excelente prognóstico. O tratamento pode incluir cirurgia conservadora da mama, mastectomia; biópsia de linfonodo sentinela e radiação da mama. Embora o tratamento também proporcione dor e desconforto, não se configura como um diagnóstico de câncer de mama. As mulheres se sentiram confusas quanto ao diagnóstico e quatro em cada seis nunca tinham ouvido falar sobre o carcinoma ductal in situ. Elas não estavam familiarizadas com a doença e referiam-se a si próprias como pacientes com câncer de mama nas entrevistas. O fardo psicológico foi instaurado pela defasagem na comunicação, gerando ansiedade e pânico (Mertz, B. G., Duriaud, H. M., Kroman, N., \& Andersen, K. G., 2017).

Em outras palavras, o momento de comunicar o diagnóstico pode ser sucedido da vulnerabilidade psicológica severa, o que aponta para necessidade do encaminhamento para um psicólogo. No entanto, toda equipe multidisciplinar deve criar um vínculo para auxiliar no alívio do sofrimento, orientar sobre os sintomas clínicos e entender o que a patologia representa para mulher, uma vez que, uma parcela considerável das pacientes que sentem dor e angústia, não fala ou pede orientação a respeito, principalmente as pacientes onde esses sintomas são predominantes, as idosas (Mertz, B. G. et al., 2017; Smith, T. G. et al., 2019).

No câncer de mama, a incapacidade associada à dor é ocasionada principalmente pela cinesiofobia, entretanto, uma correlação entre a catastrofização (processo negativo de pensamento focado nas sensações excessivas de dor) e crenças relacionadas à doença também está presente. A catastrofização influencia fatores psicológicos que podem impactar a experiência da dor, o que foi demonstrado pelos autores ao comparar as avaliações de intensidade da dor de maior escore com os sintomas de depressão (Tometich, D. B. et al., 2018; Van Der Gucht, E. et al., 2020).

A saber, os problemas de pensamento coincidem com as evidências que apontam a qualidade de vida e o impacto funcional dos sintomas cognitivos, subjetivos e objetivos relacionados ao câncer e seu tratamento. Portanto, o cuidado individualizado que atenda às necessidades emocionais e mentais dos pacientes com câncer pode resultar na melhora do controle da dor e da capacidade funcional (Fischer, D. J. et al., 2010; Tometich, D. B. et al., 2018; Van Der Gucht, E. et al., 2020).

Cada paciente maneja a dor de uma forma pessoal. Opta-se por relaxar, ficar sozinha e deitada ou em se manter mais ativa. De maneira geral, a dor aumenta a consciência do tempo e a sensação de estar presente. Consequentemente, a relação das mulheres com o tempo muda, trazendo a sensação de finitude e mortalidade. Sob o mesmo ponto de vista, ao final do tratamento pode ocorrer uma dor existencial concatenada com a solidão, em virtude de não ter mais as consultas regulares que proporcionam o acompanhamento rotineiro da equipe e o convívio com outras pacientes. Logo, as mulheres se sentem à mercê por precisar interpretar os sinais de dor por conta própria, gerando ansiedade e preocupação com a possibilidade da recidiva (Andersen, $\mathrm{C}$. et al., 2014; Hellerstedt-Börjesson, S., et al., 2015).

Certamente, a autoestima e autoimagem são afetadas pelo câncer de mama e o tratamento, uma vez que a mama é um órgão símbolo da feminilidade e maternidade. Assim sendo, o suporte emocional é importante para diminuir o humor depressivo e favorecer o controle da dor, já que um nível mais alto de controle da dor está associado com menor gravidade e níveis mais baixos de humor deprimido. Deve-se mencionar que também há uma forte relação entre os fatores que medem a saúde mental, como otimismo, resistência, desenvoltura aprendida, locus de controle, domínio, autoestima e autoeficácia, aceitação da deficiência e habilidades sociais. Além disso, o senso de coerência tem uma forte associação negativa com ansiedade, raiva, 
desmoralização, hostilidade, desesperança, depressão, estressores percebidos e transtorno de estresse pós-traumático (Cho, S., Zunin, I., Chao, P. J., Heiby, E. M., \& McKoy, J., 2012; Schrier, M. et al., 2012).

Em síntese, para a dor emocional os profissionais precisam desenvolver uma boa comunicação, incentivar a autoestima e o autocuidado. Um ensaio clínico randomizado que avaliou a efetividade de estratégias de autocuidado, demonstrou que os exercícios de respiração guiados e as faixas de relaxamento muscular progressivas foram mais úteis em relação às outras opções. A Interferência de dor e fadiga e a sensação da perda dos papéis sociais foi estatisticamente menor para o grupo que realizou as estratégias, se comparado ao grupo controle. Não só isso, mas também ocorreu a diminuição dos níveis de náusea e ansiedade no grupo que assumiu as medidas do autocuidado (Stoerkel, E. et al., 2018).

\section{Frustração Relacionada à Perda dos Papéis Sociais e a Contribuição da Rede de Apoio no Enfrentamento da Dor Social}

Por último, com o objetivo de descrever a dor social das pacientes com câncer de mama, a terceira categoria apresentouse com 25 registros. Quanto ao nível de evidência, os artigos se distribuíram como 1 com nível alto, 4 com nível moderado e 1 com nível baixo.

Ainda que seja a categoria com o menor número de achados entre os 14 artigos, denota-se como a mais notável no âmbito qualitativo. Inferimos que o baixo número pode estar relacionado com uma negligência dos profissionais ao pensar no contexto social.

O fim das responsabilidades origina uma tristeza profunda. Quando a assalariada não pode mais trabalhar ou a dona de casa tem que entregar todas as suas atividades de cuidar da família para os outros, os sentimentos de inutilidade e humilhação são despertados. Mudanças dramáticas acontecem na vida das mulheres tornando-as cada vez. Em contrapartida, a dor pode evidenciar o desejo de estar mais sozinha e isolada. A dor existencial se apresenta como uma sensação de estar completamente vulnerável e sozinha com pensamentos de aniquilação, além de ser um processo doloroso que contribui para a consciência da fragilidade humana, como também a reflexão sobre as pessoas que são significativas (Hellerstedt-Börjesson, S., et al., 2015; Saunders, 2005).

Porém, essa percepção pode ser afetada e prejudicada pela exacerbação da dor física, pois as pacientes que sentiam mais dor, reconheceram o seu temperamento e a paciência com a família serem impactados ao perceber uma irritabilidade constante e a dificuldade de lidar com as crianças. Não obstante, a dificuldade de se relacionar com as crianças não está associada somente à dor física, do mesmo modo, as mulheres percebem que os seus filhos podem perder a referência e a presença da figura materna por conta do câncer (Hellerstedt-Börjesson, S., et al., 2015; Mertz, B. G. et al., 2017).

A sensação de invalidez relacionada ao tratamento que ocasiona a renúncia dos papéis sociais é a fonte principal de frustração. No papel de companheira, as mulheres sexualmente ativas se sentem muito prejudicadas pela cirurgia, o tratamento e a terapia hormonal da doença. Os profissionais de saúde reconhecem que há fraquezas na gestão de questões de saúde sexual, refletindo diretamente no estigma e na dificuldade em discutir questões sexuais também vivenciadas no tratamento do câncer. Como por exemplo, ao realizar a reconstituição da mama, as mulheres se sentem razoavelmente satisfeitas, mas concordam com o fato de que existe um desconforto ao notar que a "nova" mama não se parece com a mama natural (Andersen, C. et al., 2014; Ferraz, V. S. et al., 2020; Mertz, B. G. et al., 2017).

O pilates foi apontado como um método benéfico para a sexualidade da mulher com câncer ao assinalar o aumento da pressão perineal e da consciência corporal relacionado aos músculos do assoalho pélvico, e consequentemente uma melhora geral na qualidade de vida. Dessa forma, para fornecer apoio social apropriado, os profissionais de saúde podem se aprofundar nas práticas integrativas e complementares para ajudar as pacientes a aprender maneiras eficazes de expressar as suas necessidades (Cho, S., et al. 2012; Ferraz, V. S. et al., 2020). 
A necessidade de frequentar um grupo com outras pacientes para compartilhar pensamentos e sentimentos reflete o amparo mútuo e a força de vontade, apesar da sensação de dor e desconforto. Isto posto, sugere que os sintomas são mais bem tratados durante o tratamento do que posteriormente, pelo fato de acontecer a identificação e interação com um grupo (Andersen, C. et al, 2014; Cho, S. et al., 2012; Mertz, B. G. et al., 2017; Smith, T. G. et al., 2019).

O cônjuge ou companheiro são a principal fonte de apoio social, seguido por familiares e amigos. Quando as mulheres pensavam sobre tudo que estavam passando, ter o apoio de alguém foi coeso com a sensação de diminuição da dor com o aumento da força para seguir em frente. Então, o ato de demonstrar diretamente as necessidades à equipe multidisciplinar e principalmente às pessoas significativas no contexto interpessoal, concebe um esforço assertivo para obter o apoio social desejado. Apesar disso, as pacientes podem não demonstrar diretamente, mas indiretamente através da postura corporal e da expressão facial. Essa busca indireta de apoio social geralmente não tem sucesso (Cho, S. et al., 2012; Hellerstedt-Börjesson, S., et al., 2015).

Em suma, toda a experiência das pacientes inclui ansiedade, depressão e medo; preocupação com a família; perda dos papéis sociais e muitas vezes a necessidade de encontrar algum significado na situação, alguma realidade mais profunda em que confiar. Portanto, cada experiência é individual e a concepção de dor deve ser ampliada, para o conceito de dor total, definido como o complexo de elementos físicos, emocionais, sociais e espirituais (Saunders, 2005).

\section{Conclusão}

Consideramos que o manejo da dor é um caminho em constante evolução. Por isso, entender qual é a vivência da dor de mulheres portadoras de câncer de mama, torna-se relevante, principalmente quando conseguimos distinguir as diferentes perspectivas da percepção dessas mulheres em relação à dor. Ao longo do estudo, entendemos ter sido primordial a compreensão, num contexto mais amplo, que não se limitou somente ao cenário nacional.

O presente estudo demonstrou um destaque para a valorização da dor física, tal fato, é um retrato de uma cultura de cuidado amparado no modelo biomédico e na medicalização. Em contrapartida, a dor emocional/mental e social/familiar foram menos contextualizadas, ao passo que a dor espiritual não foi citada, levando-nos a pensar que o conceito de dor total não pode ser negligenciado em mulheres portadoras de câncer de mama. Foi oportuno observar que, diferentes métodos não farmacológicos podem ser utilizados no enfrentamento da dor, estimulando a autoestima e a qualidade de vida, bem como menos uso de métodos farmacológicos.

Caracterizam-se como limitações do estudo, a falta de descritores mais específicos para delimitação da estratégia de busca e a ausência de recursos para financiar artigos substanciais não disponibilizados gratuitamente. Iremos encaminhar para a organização responsável pelo DeCS e MesH Terms uma sugestão de inclusão dos descritores: dor física, dor emocional, dor social e dor espiritual.

Conclui-se que novos estudos que visem ressaltar os aspectos da dor espiritual são necessários para alcançar o cuidado genuinamente integral das pacientes, e quanto mais informações forem pesquisadas e analisadas, maior será a eficácia do manejo da dor. Logo, sugerimos para a academia o desenvolvimento de pesquisas que estejam relacionadas com a espiritualidade dos pacientes, mensurando a importância de instituições e profissionais de saúde dispostos a promover espaços onde o paciente possa expressar a fé, seja ela qual for; e estudos que possam identificar a profundidade dessa temática para além da religião.

\section{Referências}

Amraoui, J., Pouliquen, C., Fraisse, J., Dubourdieu, J., Guzer, S. R. D., Leclerc, G., Forges, H., Jarlier, M., Gutowski, M., Bleuse, J. P., Janiszewski, C., Diaz, J., \& Cuvillon, P. (2018). Effects of a hypnosis session before general anesthesia on postoperative outcomes in patients who underwent minor breast cancer surgery: the HYPNOSEIN randomized clinical trial. JAMA Network Open, Montpellier, França. v. 1, n. 4, p. e181164-e181164.

Andersen C., Rørth M., Ejlertsen B., \& Adamsen L. (2014). Exercise despite pain - breast cancer patient experiences of muscle and joint pain during adjuvant chemotherapy and concurrent participation in an exercise intervention. European Journal of Cancer Care, Copenhagen, Dinamarca. v.23, p. $653-667$. 
Araújo, I. B. S., Leite, C. B., Amorim, T. O., Silva, A. N. S., Fernandes, R. S. Q. \& Carmo, M. S. (2018). Câncer de mama em homens. Revista de Investigação Biomédica, São Luís, Brasil. v.10, n.3, p. 272-279.

Bardin, L. (2011). Análise de Conteúdo. Edições 70, São Paulo, Brasil.

Brasil. Ministério da Saúde. (2016). Protocolos da Atenção Básica: Saúde das Mulheres. Instituto Sírio-Libanês de Ensino e Pesquisa, Brasil, Brasília. p. 187.

Cho, S., Zunin, I., Chao, P. J., Heiby, E. M., \& McKoy, J. (2012). Effects of pain controllability and discrepancy in social support on depressed mood among patients with chronic pain. International Journal of Behavioral Medicine, Hawaii, Estados Unidos da América. v. 19, n. 3, p. $270-279$.

Costa, C. A., Santos, C., Alves, P., \& Costa. (2007) Dor oncológica. Revista Portuguesa de Pneumologia, Portugal, Lisboa. v. 13 , n. 6, p. 855-867.

Couto, M. S. A., Guerra, M. R., Firme, V. A. C., \& Teixeira, M. T. B. (2017). Comportamento da mortalidade por câncer de mama nos municípios brasileiros e fatores associados. Revista Panamericana de Salud Pública, Minas Gerais, Brasil. v.41, 168.

Ferraz, V. S., Cruz, A. D. F. C., Ferreira, M. A. M., Burke, T. N., Júnior, S. A. O., Christofoletti, G., \& Pegorare, A. B. G. S. (2020). Effect of the Pilates Method on Sexual Function, Pelvic floor Muscle Strength and Quality of Life of Breast Cancer Survivors. Revista Brasileira de Cancerologia, Mato Grosso do Sul, Brasil.

Fischer, D. J., Villines, D., Kim, Y. O., Epstein, J. B., \& Wilkie, D. J. (2010). Anxiety, depression, and pain: differences by primary cancer. Supportive Care in Cancer, Chicago, Estados Unidos da América. v. 18, n. 7, p. 801-810.

Hellerstedt-Börjesson, S., Nordin, K., Fja“llskog, M. L., Holmstro“m, I. K., \& Arving, C. (2015). Women with breast cancer: experience of chemotherapyinduced pain: triangulation of methods. Cancer Nursing, Uppsala, Suiça. v. 38, n. 1, p. 31-39.

INCA. (2020b) Câncer de mama. Instituto Nacional de Câncer, Rio de Janeiro, Brasil.

INCA. (2020b). Estimativa 2020: incidência de câncer no Brasil. Instituto Nacional de Câncer, Rio de Janeiro, Brasil.

Mertz, B. G., Duriaud, H. M., Kroman, N., \& Andersen, K. G. (2017). Pain, sensory disturbances, and psychological distress among Danish women treated for ductal carcinoma in situ: an exploratory study. Pain Management Nursing, Copenhagen, Dinamarca. v. 18, n. 5, p. 309-317.

Oliveira, D. S. S., Roque, V. A., \& Maia, L. F. S. (2019). A dor do paciente oncológico: as principais escalas de mensuração. Revista Recien, Brasil, São Paulo. v. $9(26): 40-59$

Oxford Centre for Evidence-Based Medicine. (2013). Levels of evidence. Oxford, Inglaterra.

Park, K. U., Kyrish, K., Terrel, J., Yi, M., Caudle, A. S., Hunt, K. K., Kuerer, H. M., Bedrosian, I., Thompson, A., \& Snyder, S. M. (2019). Surgeon perception versus reality: opioid use after breast cancer surgery. Journal of Surgical Oncology, Texas, Estados Unidos da América. v. 119, n. 7, p. 909-915.

Raja, S. N., Carr, D. B., Cohen, M., Finnerup, N. B., Flor, H., Gibson, S., Keefe, F. J., Mogil, J. S., Ringkamp, M., Sluka, K. A., Song, X. J., Stevens, B., Sullivan, M. D., Tutelman, P. R., Ushida, T., \& Vader, K. (2020). Definição revisada de dor pela Associação Internacional para o Estudo da Dor: conceitos, desafios e compromissos. Sociedade Brasileira para o Estudo da Dor, tradução, Brasil, Brasília.

Samuel, C. A., \& Faithfull, S. (2014). Complementary therapy support in cancer survivorship: a survey of complementary and alternative medicine practitioners' provision and perception of skills. European Journal of Cancer Care, Guildford, Reino Unido. v. 23, n. 2, p. $180-188$.

Santana, M. C., Rippel, J. R. S. \& Fortes, R. C. (2020). Boletim informativo para prevenção e diagnóstico precoce do câncer de mama. Health Residencies Journal, Brasília, Brasil. v. 1, n. 6, p. 54-62.

Saunders, D. C. (2005). Watch with me: inspiration for a life in hospice care. Observatory Publications. Lancaster, Reino Unido.

Schrier, M., Amital, D., Arnson, Y., Rubinow, A., Altaman, A., Nissenabaum, B., \& Amital, H. (2012). Association of fibromyalgia characteristics in patients with non-metastatic breast cancer and the protective role of resilience. Rheumatology international, Jerusalém, Israel. v. 32, n. 10, p. 3017-3023.

Smith, T. G., Troeschel, A. N., Castro, K. M., Arora, N. K., Stein, K., Lipscomb, J., Brawley, O. W., McCabe, R. M., Clauser, S. B., \& Ward, E. (2019). Perceptions of patients with breast and colon cancer of the management of cancer-related pain, fatigue, and emotional distress in community oncology. Journal of Clinical Oncology, Estados Unidos América. v. 37, n. 19, p. 1666.

Sousa, L. M. M., Vieira, C. M. A. M., Severino, S. S. P., \& Antunes, A. V. (2017). Metodologia de revisão integrativa da literatura em enfermagem. Revista Investigação em Enfermagem, Porto, Portugal. n. 21, p. 17.

Stoerkel, E., Bellanti, D., Paat, C., Peacock, K., Aden, J., Setlik, R., Walter, J., \& Inman, A. (2018). Effectiveness of a self-care toolkit for surgical breast cancer patients in a military treatment facility. The Journal of Alternative and Complementary Medicine, Texas, Estados Unidos da América. v. $24, \mathrm{n} .9-10$, p. 916-925.

Tometich, D. B., Mosher, C. E., Hirsh, A. T., Rand, K. L., John, S. A., Matthias, M. S., Outcalt, S. D., Schneider, B. P., Minas, L., Storniol, A. M., Newton, E. V., \& Miller, K. (2018). Metastatic breast cancer patients' expectations and priorities for symptom improvement. Supportive Care in Cancer, Indianapolis, Estados Unidos da América. v. 26, n. 11, p. 3781-3788.

Van Der Gucht, E., Dams, L., Meeus, M., Devoogdt, N., Beintema, A., Penen, F., Hoelen, W., Vrieze, T., \& Groef, A. (2020). Kinesiophobia contributes to pain-related disability in breast cancer survivors: a cross-sectional study. Supportive Care in Cancer, p. 1-8.

World Health Organization, WHO. (2020). WHO report on cancer: setting priorities, investing wisely and providing care for all. Geneva, Suíça. 